\title{
REQUERIMIENTOS EN ASESORÍA CONTABLE Y TRIBUTARIA EN LAS PYMES DE MANTA, ECUADOR
}

\section{REQUIREMENTS IN ACCOUNTING AND TAX ADVICE IN SMES IN MANTA, ECUADOR}

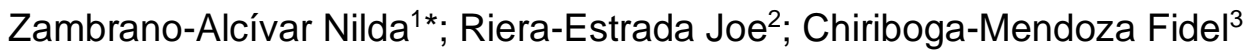 \\ ${ }^{1}$ Docente de la Universidad Laica Eloy Alfaro de Manabí, ULEAM. Manta, Ecuador \\ ${ }^{2}$ Docente de la Universidad Laica Eloy Alfaro de Manabí, ULEAM. Manta, Ecuador \\ ${ }^{2}$ Docente de la Universidad Laica Eloy Alfaro de Manabí, ULEAM. Manta, Ecuador
}

*Correo: nilzam190293@hotmail.com

\begin{abstract}
Resumen
El proyecto para evaluar la necesidad de una empresa que realice asesoría contable y tributaria en la ciudad de Manta, se lleva a cabo con la finalidad de contribuir al desarrollo sectorial de las Pymes que operan en este cantón; el estudio se enfoca directamente en analizar las necesidades latentes de la mayoría de empresas de esta categoría en temas contables y tributarios, para de esta manera analizar la situación de las pymes que requieren los servicios de asesoría contable y tributaria, mediante un análisis de mercado según las cinco fuerzas de Porter. La investigación se llevó a cabo mediante la metodología bibliográfica documental y de campo, utilizando herramientas como la entrevista y la encuesta, orientadas a los gerentes de las firmas de consultoría contable ya existentes y a las Pymes de la ciudad de Manta respectivamente. Los resultados arrojados por la investigación realizada concluyen en la necesidad del proyecto de creación de una empresa asesora contable y tributaria que oferte sus servicios a las pymes de la ciudad de Manta, contando con alta aceptación dentro de este sector.
\end{abstract}

Palabras clave: contabilidad, tributación, empresa, pymes, fuerzas de Porter.

\begin{abstract}
The project to evaluate the need for a company that performs accounting and tax advice in the city of Manta, is carried out with the aim of contributing to the sector development of the SMEs that operate in this canton; The study focuses directly on analyzing the latent needs of most companies in this category in accounting and tax issues, in order to analyze the situation of SMEs that require accounting and tax consulting services, through a market analysis according to Porter's five forces. The research was carried out using the documentary and field bibliographic methodology, using tools such as the interview and the survey, aimed at the managers of the existing accounting consulting firms and the SMEs in the city of Manta, respectively. The results of the research carried out conclude on the need for the project to create an accounting and tax advisory company that offers its services to SMEs in the city of Manta, with high acceptance within this sector.
\end{abstract}

Keywords: accounting, taxation, company, SMEs, Porter's forces.

Información del manuscrito:

Fecha de recepción: 28 de julio de 2017

Fecha de aceptación: 13 de diciembre de 2017

Fecha de publicación: 10 de enero de 2018 


\section{Introducción}

A nivel global es considerado que las pequeñas y medianas empresas son un factor fundamental en la generación de empleo y en el crecimiento económico de un país, lo que demuestra que existe una estrecha relación entre el grado de desarrollo de las PYMES y el nivel del ingreso per cápita del estado. Ésta, entre otras razones, justifica la preocupación permanente del sector privado y de las autoridades económicas por impulsar su crecimiento y consolidación (Salavou et al., 2004).

Uno de los principales problemas que tienen las PYMES, es que presentan un nivel bajo de ingreso, razón por la cual no cuentan con una estabilidad económica que les permita sostenerse en su actividad comercial. A lo que se suma que las condiciones de acceso al crédito son poco competitivas a nivel en la mayoría de los países de la región; por causa de los altos costos de crédito con los que cuentan estas empresas, además los plazos ofertados son cortos y las exigencias en garantías son elevadas. Interviene también y es muy común, la falta de confianza de los bancos respecto a los proyectos de estas empresas (Hussinger, 2010).

Pero no sólo es por la falta de apoyo económico, sino que también interviene el deficiente control que presentan la mayoría de estas empresas; las más comunes, son la falta de transparencia contable, el costo de obtención de la información adecuada. El poco conocimiento de los sistemas básicos de control de gestión da lugar a una administración que presenta fraudes, desconocimiento del verdadero estado financiero de la empresa, gastos innecesarios, mal manejo de los inventarios (Arenas et al., 2009).

Muchas PYMES en Ecuador aún tienen problemas al escoger e implementar exitosamente un sistema contable de acuerdo a sus necesidades. A pesar de que la mayoría de empresas cuenta con algún tipo de sistema computarizado que ayude en las tareas contables, uno de los factores comunes en el ámbito empresarial de las PYMES es la falta de estandarización de sistemas contables o software contable que permitan contar con información instantánea y al día. Es decir, hay muchas ofertas, poca 
estandarización, poco soporte, y mucha confusión (Grupo Enroke, 2013).

Por lo antes mencionado se puede conocer que la ausencia de sistemas eficaces para llevar una correcta contabilidad genera que en un sinnúmero de ocasiones existan altos riesgos, donde se involucra la rentabilidad del negocio, ya sea por una asesoría inadecuada o en el peor de los casos por obviar la colaboración de un asesor especializado. Por lo antes mencionado se puede conocer que la ausencia de sistemas eficaces para llevar una correcta contabilidad genera que en un sinnúmero de ocasiones existan altos riesgos, donde se involucra la rentabilidad del negocio, ya sea por una asesoría inadecuada o en el peor de los casos por obviar la colaboración de un asesor especializado.

Ferrin-Schettini et al. (2016) mencionan que los sistemas de contabilidad computarizada agilitan de una manera técnica el proceso de elaboración de estados financieros con su respectivo proceso contable, según una investigación realizada en una empresa productora de tagua en la ciudad de Manta. No obstante, es una situación palpable en la ciudad y en muchas localidades del Ecuador observar negocios clausurados 0 con limitantes en su desarrollo económico, lo cual se produce en su mayoría por una economía desorganizada, la falta de control con exactitud en sus movimientos transaccionales y el incumplimiento de disposiciones legales y tributarias, causadas generalmente por los altos índices de desconocimiento 0 falta de capacitación en el ámbito contable.

De acuerdo a Jaen \& Valverde (2013) los directivos de las PYMES, en la mayoría de las ocasiones, no priorizan la automatización de sus procesos financieros-contables, ya que la inversión es altamente costosa y requiere de un tiempo mínimo de seis meses para la implementación de un sistema adecuado que se adapte a sus necesidades. Otros optan por adquirir sistemas informáticos estándares que en la mayoría de las veces no se acoplan al modelo de la organización ni satisfacen los requerimientos. Es por ello que se propone una investigación que pueda demostrar el punto de vista de este sector económico de la ciudad y 
el enfoque se le da al aspecto contable dentro de sus procesos administrativos, legales y tributarios. Determinando así la funcionalidad de las empresas al implementar sistemas de esta naturaleza.

El estudio de factibilidad para la creación de una firma de asesoría que oferte un portafolio de servicios contables y tributarios a pequeñas y medianas empresas (PYMES) de la ciudad de Manta, se lleva a cabo en base a un estudio en el cual se detalla la realidad del entorno de este sector económico de la ciudad.

\section{Metodología}

\subsection{Modalidad de la investigación}

El proyecto de investigación por ser de naturaleza científica-exploratoria, se basó en el estudio de campo con la obtención de fuentes primarias y a su vez en la investigación bibliográfica como fuente de datos secundaria, complementada con ello un estudio de análisis y profundidad. Se explica el uso y aplicación de cada modalidad:

De campo.- La investigación es de campo pues es necesario desarrollar un estudio que permita obtener información de las Pymes y la realidad de las mismas en base al tema de estudio, para ello es preciso acudir al lugar de los hechos y recolectar datos por medio de herramientas eficaces.

Bibliográfica.- Por otro lado, se considera una investigación bibliográfica porque se recurre al uso de medios de información documental tales como libros, informes, revistas, web entre otros., para alcanzar así un sustento comprensible de la terminología y las variables explicadas en el marco teórico y conceptual del plan.

\subsection{Nivel de investigación}

Correlacional.- El estudio presenta en primera instancia un nivel de este tipo, ya que a través de la manipulación y relación de las variables se pudo generar conocimiento inmediato de los fenómenos y hechos analizados en este apartado. En este caso al generar comparación de una variable con otra se establece una hipótesis que podrá ser 0 no demostrada a lo largo del estudio.

Explicativo.- Por otra parte se llega a un nivel explicativo, pues a través del estudio de campo se alcanzaron resultados que pueden generar opciones diversas de solución al 
problema planteado. Es aquí donde se explica el desarrollo de la propuesta como base esencial del proyecto.

Cuantitativa y cualitativa.- la investigación se considera de enfoque mixto (cualitativa y cuantitativa), puesto que la información inicial ha sido medida a través de un análisis para llegar a conclusiones breves, por otro lado se considera cuantitativa pues al desarrollar un estudio de campo, existe información que debe ser procesada, analizada e interpretada para concluir con datos exactos.

\subsection{Población y muestra}

La población considerada para el estudio consistió en las pequeñas y medianas empresas situadas en la ciudad de Manta, llegándose a conocer que en la actualidad existe un número aproximado de 1500 Pymes, según datos proporcionados por El Banco Solidario (Área de Negocios).

\section{Muestra}

Para este caso se recurrió al uso del muestreo probabilístico aplicando el método aleatorio estratificado, tomando en cuenta que se trabajó con dos características de la población, como son las pequeñas y medianas empresas, de las cuales se determinó un número de encuestas para cada una según la conveniencia del plan. La muestra seleccionada es de 20 Pymes.

Para la recolección de información se empleó la encuesta como técnica apta para el estudio, apoyándose mediante la estructuración de un cuestionario de preguntas dirigidas a los dueños de las Pymes de la ciudad de Manta.

\subsection{Procesamiento y análisis de datos}

El procesamiento y análisis de datos representa una labor de gran importancia dentro del plan investigativo, pues de ello depende el análisis correcto de los resultados obtenidos en la investigación. Para ello se utilizaron las herramientas tecnológicas necesarias por medio de una tabulación y representación gráfica de claridad y entendimiento a mediante Microsoft Excel.

\section{Resultados y discusión}

La firma "MAR Consultoría y Auditoría" fue una de las empresas a las cuales se acudió para obtener información acerca de su desempeño como entes asesores de 
Pymes en la ciudad de Manta, llegándose a conocer que esta firma lleva 25 años en el mercado, cuentan que durante su experiencia en asesoría contable hacia el sector de las Pymes han notado que este tipo de empresas se desarrollan de forma limitada o con lentitud, diariamente se encuentran con grandes inconvenientes tales como, procesos contables no bien definidos pues prefieren contratar contadores autónomos e independientes que no muestran alto compromiso y seriedad para alcanzar los mejores resultados. Los servicios por los cuales acuden las Pymes a esta firma guardan relación principalmente con las declaraciones de Impuesto a la Renta y Formularios de retenciones. La mayor anécdota para esta firma contable y su relación con las pequeñas y medianas empresas ha sido encontrarse con talento humano no capacitado dentro de las empresas en crecimiento, para ser específicos el personal del área contable en su mayoría desconoce de las NIIF, lo cual deriva en incongruencias en los registros contables y problemas tributarios.
Otra de las firmas contables donde se aplicó la entrevista fue en "AUDICONT"; su gerente expresa que su experiencia con las Pymes se centra en brindar un asesoramiento adecuado, además da a conocer que estas empresas carecen de buenas prácticas tributarias, presentan inconvenientes tributarios, legales, optan por una asesoría inadecuada y sus actividades las centran en el intercambio de bienes y servicios olvidando la importancia de mantener los registros de estos movimientos para efectivizar su rentabilidad. Los servicios de mayor demanda por parte de las Pymes en esta firma son las declaraciones tributarias, teneduría de libros y manejo de facturación. Una anécdota muy particular que se destaca de esta entrevista consiste en que las Pymes son constituidas legalmente por socios que pertenecen al mismo entorno familiar por mero trámite, sin considerar la importancia del capital contable para inversiones presentes y futuras, presentando en corto plazo problemas de liquidez.

Por parte de la empresa "Sociedad de Auditores y Contadores S.A VICJAV", se pudo conocer que su 
experiencia para con las Pymes se ha basado en el manejo de microcréditos, cierres de balances, temas tributarios, laborales y toma de decisiones. Su gerente detalla que los problemas que surgen con mayor frecuencia en este tipo de negocios en crecimiento, corresponden a falta de liquidez, de financiamiento, falta de control en las finanzas y por ende continuos inconvenientes en temas tributarios. Comentan que los servicios que contratan las Pymes se engloban en: Declaraciones tributarias, Anexos y Devolución de IVA. Por último, el entrevistado cuenta que su mayor anécdota trabajando en el campo de la asesoría fue concebida cuando llegaron a brindar sus servicios a una empresa chilena, de lo cual le llamó mucho la atención que, a diferencia de las Pymes locales, estas son organizadas, muy exigentes y manejan control total en cada actividad para generar cumplimiento y alcance de metas.

En la aplicación de la encuesta se obtuvieron los siguientes resultados:

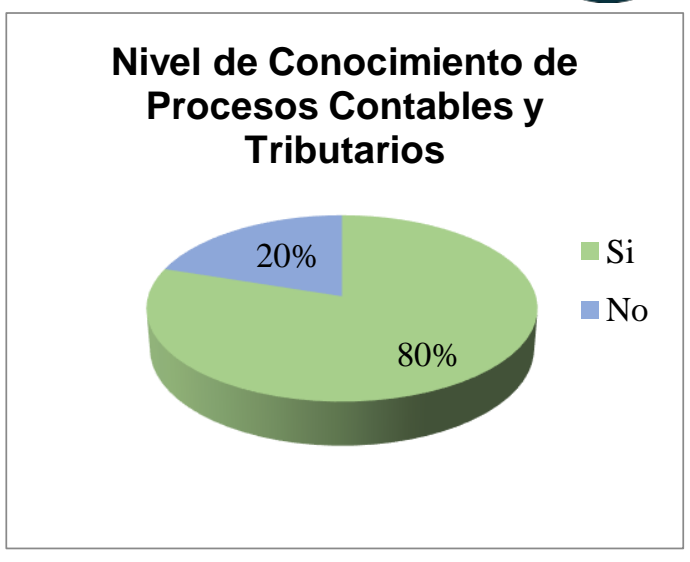

Figura 1. Grado de conocimiento en temas tributarios y contables

Aplicando la técnica de la encuesta al sector Pymes de la ciudad de Manta y abordando acerca del nivel de conocimientos en cuanto a procesos contables y tributarios, se determinó que el $80 \%$ de los encuestados dice tener conocimiento en el área contable, mientras que el $20 \%$ restante opina no estar capacitado en estos temas (figura 1). Por ello se requiere promover la capacitación y formación continua en esta área.

La figura 2 muestra la forma en la que las pymes llevan sus controles contables y tributarios, llegándose a conocer que el $50 \%$ realiza sus procesos contables por medio de la asesoría externa ya sea de contadores independientes o firmas contables; el $45 \%$ lleva sus controles de forma interna; mientras que el $5 \%$ 
no lleva ningún control o registro en sus finanzas.

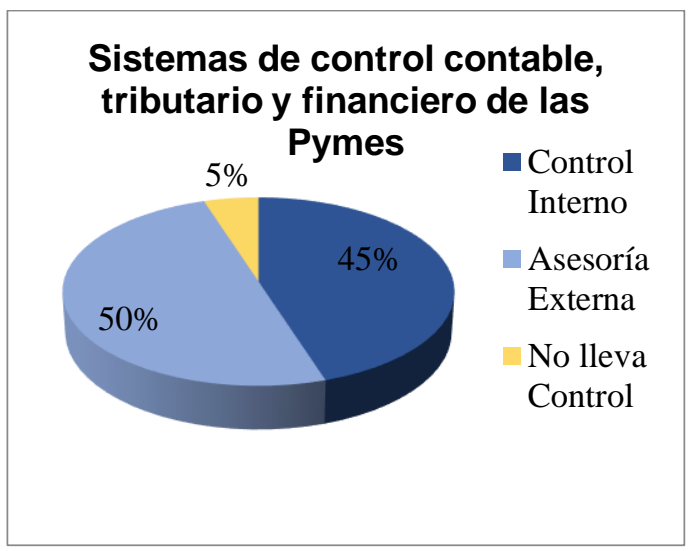

Figura 2. Formas empleadas por las pymes para realizar controles contables

Por otra parte, el $25 \%$ de las pymes reflejan inconvenientes y problemas legales. Para medir la demanda de las Pymes en cuanto a servicios de asesoría se consultó si se han realizado requerimientos de firmas contables para llevar sus procesos, obteniendo que el $80 \%$ opina que si ha solicitado este tipo de asesoría externa; mientras que el $20 \%$ dio a conocer que nunca ha recurrido a una firma de asesoría contable para llevar procesos tributarios 0 de finanzas. La figura 3 señala cuales son los servicios de asesoría externa demandados por las pymes.

Refiriéndose a los servicios demandados por las Pymes y su nivel de requerimiento se llega a conocer que el $50 \%$ solicita diversos servicios a la vez, entre los que sobresalen los reportes contables, las declaraciones de impuestos y las capacitaciones; por otra parte, el $30 \%$ se enfoca en contratar servicios contables solo para temas de declaración de impuestos y el $20 \%$ dice no solicitar los servicios de firmas contables.

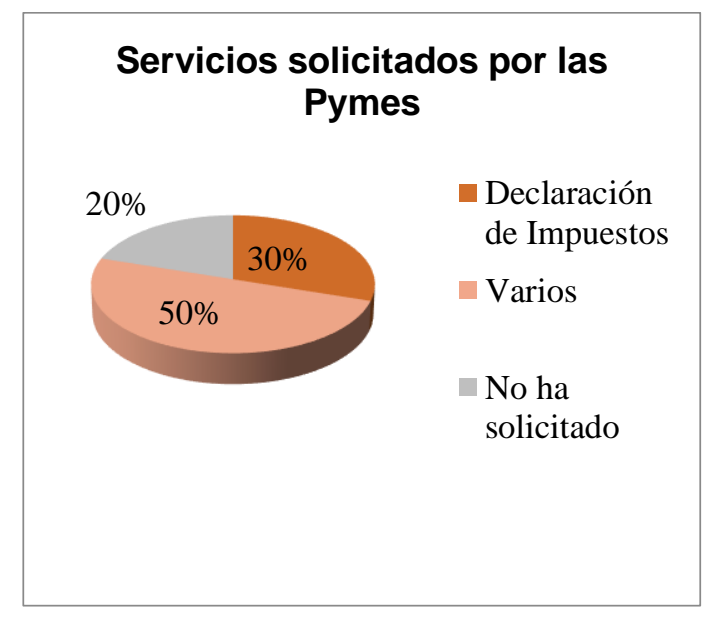

Figura 3. Servicios de asesoría externa demandados por las pymes

\subsection{Análisis de mercado}

La figura 4 presenta un análisis de mercado según las cinco fuerzas de Porter. Se evalúan los siguientes factores:

\section{a) Amenaza de Nuevos competidores (Alta):}

El posicionamiento de las firmas contables en todos los mercados ha mostrado un incremento considerable, relacionándose a la aparición de múltiples negocios de diferentes tamaños, es por esta 
razón que existe una alta amenaza de entrada de nuevos competidores dentro de la oferta de servicios contables y tributarios. Cada vez son más las empresas que con poca experiencia en el manejo de sus cuentas acceden a la tercerización de este tipo de servicios, asegurando con ello efectividad en el control de sus finanzas. El ingreso al mercado es totalmente factible, desde el punto de vista de la demanda, así como el financiamiento, puesto que, para ofrecer servicios de esta categoría, el llevar a cabo una firma contable dentro del sector presenta pocos limitantes, es decir puede existir un alto índice competitivo en la ciudad de Manta.

\section{b) Poder de Negociación de los} Clientes (Medio):

Dentro del estudio para la creación de una firma contable el poder de negociación de los clientes se considera en un rango medio, partiendo del hecho que el mercado al que se enfoca la oferta es un sector con gran necesidad en la aplicación de controles y registros contables, por ende, la relación entre la firma y quien contrae negociaciones con ella, debe regirse a través de parámetros previamente ajustados para el beneficio de ambas partes.

\section{c) Amenaza de Servicios Sustitutos (Alta):}

Existe alta amenaza de servicios sustitutos dentro del sector, esto se debe a que un gran número de profesionales en la rama contable se dedican a la oferta de servicios de esta naturaleza, bajo el tratamiento de persona natural, lo cual dificulta en diversas ocasiones que las Pymes accedan a la contratación de agencias 0 firmas especializadas donde se puede aplicar un sistema de control con mayores y mejores estudios de adaptabilidad según su modelo de negocio.

\section{d) Poder de Negociación de los Proveedores (Baja):}

El poder de negociación con los proveedores representa una baja amenaza para la firma contable que se pretende crear, considerando la periodicidad de uso de suministros, sistemas de información, datos 0 demás requerimientos de la entidad.

e) Rivalidad entre los Competidores del Sector (Media):

Existe una mediana rivalidad entre competidores del sector, la oferta de 
servicios contables y tributarios a través de entidades de personería jurídica es reducida, existen alrededor de seis empresas dedicadas a este rubro, unas con mayor experiencia tales como "DIRECSA" y "INZAMTOR CIA. LTDA.", además de las firmas con menor tiempo en el mercado como "FERNANDEZ DE CORDOBA \& ASOCIADOS CIA. LTDA.", "VICJAV" y "LEANSPIRE S.A."; dedicadas en su mayoría a la consultoría interna y externa, asesoría jurídica o financiera, gestión organizacional y capacitación.

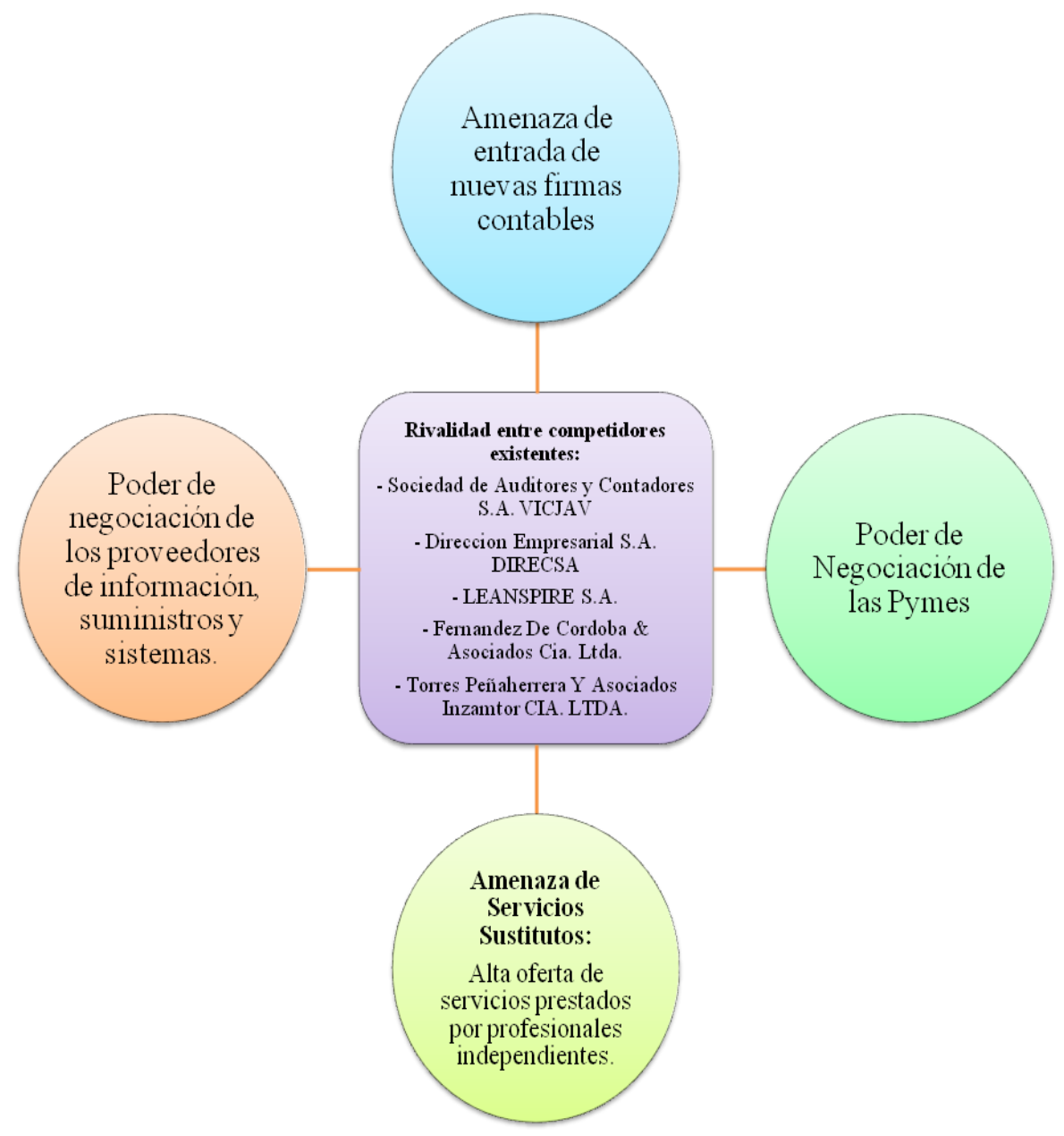

Figura 4. Fuerzas de Porter para el análisis del mercado

Tal como se indica en la interpretación de los resultados, existe una demanda considerable en servicios para el sistema de control contable y tributario de las pymes. En Manta, ya se ha demostrado la necesidad de las empresas respecto a sistemas de contabilidad 
computarizada, que permitan un manejo óptimo de los estados financieros (Ferrin-Schettini et al., 2016).

\section{Conclusiones}

Para las Pymes contar con un departamento contable representa costos operativos elevados, por esta razón necesitan contratar servicios externos de asesoría contable y tributaria a costos accesibles, que garanticen mayor productividad en el corto plazo. Con el tratamiento de datos se ha determinado que el mercado se encuentra desatendido por las empresas asesoras de la localidad, pues en su mayoría se dedican a prestar sus servicios a grandes empresas.

Los resultados arrojados por la investigación de mercados concluyen en la necesidad del proyecto de creación de una empresa asesora contable y tributaria para las pymes de la ciudad de Manta, contando con alta aceptación en este sector.

\section{Bibliografía}

Arenas, Charris, Manga, \& Ríos. (2009). Deficiencias contables de las PYMES del sector comercial. En Arenas, Charris, Manga, \& Ríos,
Deficiencias contables de las PYMES del sector comercial (pág. 144; 145). BarranquillaColombia: Universidad Simón Bolivar de Barranquilla.

Ferrin-Schettini, H., Alcívar-Mero, M., \& Mero-Mero, R. (2016). Avance tecnológico aplicando un sistema contable en la empresa Tagua Marfil del puerto de Manta. Dominio de las Ciencias, 2(1), 176-192.

Grupo Enroke. (2013). Programas Contables para PYMES. Recuperado el 04 de 08 de 2014, de http://grupoenroke.com/index. php/noticias/53-programascontables-para-pymes

Hussinger, K. (2010). On the importance of technological relatedness: SMEs versus large acquisition targets. Technovation, 30(1), 57-64.

Jaen, \& Valverde. (2013). Realidad de los procesos financieroscontables de las Pymes. Guayaquil-Ecuador: Universidad Politécnica Salesiana.

Salavou, H., Baltas, G., \& Lioukas, S. (2004). Organisational innovation in SMEs: the importance of strategic orientation and competitive structure. European journal of marketing, 38(9-10), 10911112. 